\title{
Mice lacking ghrelin receptors resist the development of diet-induced obesity
}

\author{
Jeffrey M. Zigman,, ${ }^{1}$ Yoshihide Nakano, ${ }^{1}$ Roberto Coppari, ${ }^{1}$ Nina Balthasar, ${ }^{1}$ Jacob N. Marcus, ${ }^{1}$ \\ Charlotte E. Lee, ${ }^{1}$ Juli E. Jones, ${ }^{1}$ Amy E. Deysher, ${ }^{1}$ Amanda R. Waxman, ${ }^{1}$ Ryan D. White, ${ }^{1}$ \\ Todd D. Williams, ${ }^{1}$ Jennifer L. Lachey, ${ }^{1}$ Randy J. Seeley, ${ }^{2}$ Bradford B. Lowell, ${ }^{1}$ and Joel K. Elmquist ${ }^{1,3}$ \\ 1Department of Medicine, Division of Endocrinology, Diabetes and Metabolism, Beth Israel Deaconess Medical Center (BIDMC), Harvard Medical School, \\ Boston, Massachusetts, USA. 'Department of Psychiatry, University of Cincinnati, Cincinnati, Ohio, USA. ${ }^{3}$ Department of Neurology, \\ BIDMC and Program in Neuroscience, Harvard Medical School, Boston, Massachusetts, USA.
}

\begin{abstract}
Ghrelin is the endogenous ligand for the growth hormone secretagogue receptor (GHSR; ghrelin receptor). Since its discovery, accumulating evidence has suggested that ghrelin may play a role in signaling and reversing states of energy insufficiency. For example, ghrelin levels rise following food deprivation, and ghrelin administration stimulates feeding and increases body weight and adiposity. However, recent loss-of-function studies have raised questions regarding the physiological significance of ghrelin in regulating these processes. Here, we present results of a study using a novel GHSR-null mouse model, in which ghrelin administration fails to acutely stimulate food intake or activate arcuate nucleus neurons. We show that when fed a high-fat diet, both female and male GHSR-null mice eat less food, store less of their consumed calories, preferentially utilize fat as an energy substrate, and accumulate less body weight and adiposity than control mice. Similar effects on body weight and adiposity were also observed in female, but not male, GHSR-null mice fed standard chow. GHSR deletion also affected locomotor activity and levels of glycemia. These findings support the hypothesis that ghrelin-responsive pathways are an important component of coordinated body weight control. Moreover, our data suggest that ghrelin signaling is required for development of the full phenotype of diet-induced obesity.
\end{abstract}

\section{Introduction}

Ghrelin is the endogenous ligand of the growth hormone secretagogue receptor (GHSR; ghrelin receptor) and is named for its potent growth hormone-secreting (GH-secreting) properties (1). It also has been hypothesized that ghrelin plays an important role in signaling energy insufficiency. For example, ghrelin levels rise prior to meals and following food deprivation (2-4); ghrelin administration potently stimulates feeding, while GHSR antagonists blunt feeding (4-9). Ghrelin also lowers energy expenditure and increases respiratory quotient, body weight, and adiposity (5, $6,8,10,11)$. Based on these studies, targeted deletion of ghrelin or its receptor was expected to have a significant effect on feeding behavior and body fat deposition. However, 3 recent loss-offunction studies have raised questions regarding the physiological significance of ghrelin in regulating these processes (12-14). For instance, Sun and colleagues reported an insignificant difference between ghrelin-knockout animals and wild-type controls in body weight on either standard chow or high-fat diet (HFD). They also found no differences in cumulative food intake on standard chow or body weight change and food intake in response to re-exposure to food following a fast (12). Wortley and colleagues reported only slight differences between ghrelin-knockout animals and wild-type controls in body composition upon acute exposure to HFD (14). Finally, Sun and colleagues reported that their GHSR-knockout

Nonstandard abbreviations used: CLAMS, Comprehensive Lab Animal Monitoring System; DEXA, dual-energy x-ray absorptiometry; GH, growth hormone; GHSR, growth hormone secretagogue receptor, ghrelin receptor; HFD, high-fat diet; icv, intracerebroventricular(ly); NMR, quantitative nuclear magnetic resonance; TBC, transcriptional blocking cassette.

Conflict of interest: The authors have declared that no conflict of interest exists.

Citation for this article: J. Clin. Invest. 115:3564-3572 (2005).

doi:10.1172/JCI26002. animals, in comparison with wild-type controls, had only a modest decrease in body weight when maintained on standard chow (13).

Here we present data collected from experiments with a novel GHSR-null mouse model. We found that, in contrast to previous studies, lack of GHSR does significantly affect body weight homeostasis, both in animals fed standard chow and in animals challenged with HFD.

\section{Results}

Generation of GHSR-null mice. GHSR-null mice were created by inserting a loxP-flanked transcriptional blocking cassette (TBC) into a putative intron located downstream of the transcriptional start site and upstream of the translational start site of the murine Ghsr gene (Figure 1). The TBC includes the following elements (in the order listed): a splice acceptor site from the mouse engrailed 2 gene (En-2) followed by an SV40 poly(A) signal, an SV40 enhancer followed by a neomycin resistance gene and $2 \mathrm{HSV}$-TK poly(A) signals, a synthetic poly(A) signal/transcriptional pause signal, and another synthetic poly(A) signal followed by a Myc-associated zinc finger protein-binding site (15-17). This nonstandard, "knockout" approach, in which the Ghsr locus was modified by the addition of a loxp-flanked TBC rather than by the removal of a critical exon or exons, was used in order to enable Cre recombinase-mediated reactivation of GHSR expression in future experiments.

GHSR-null mice (harboring 2 copies of the recombinant Ghsr allele) and their littermates, which included heterozygotes (harboring 1 copy of the recombinant Ghsr allele and 1 copy of the wild-type Ghsr allele) and wild-type mice (harboring 2 copies of the wild-type Ghsr allele), were viable and were born with the expected Mendelian distribution upon mating of heterozygotes. These respective genotypes were confirmed by Southern blot analysis of genomic DNA (Figure 1). 

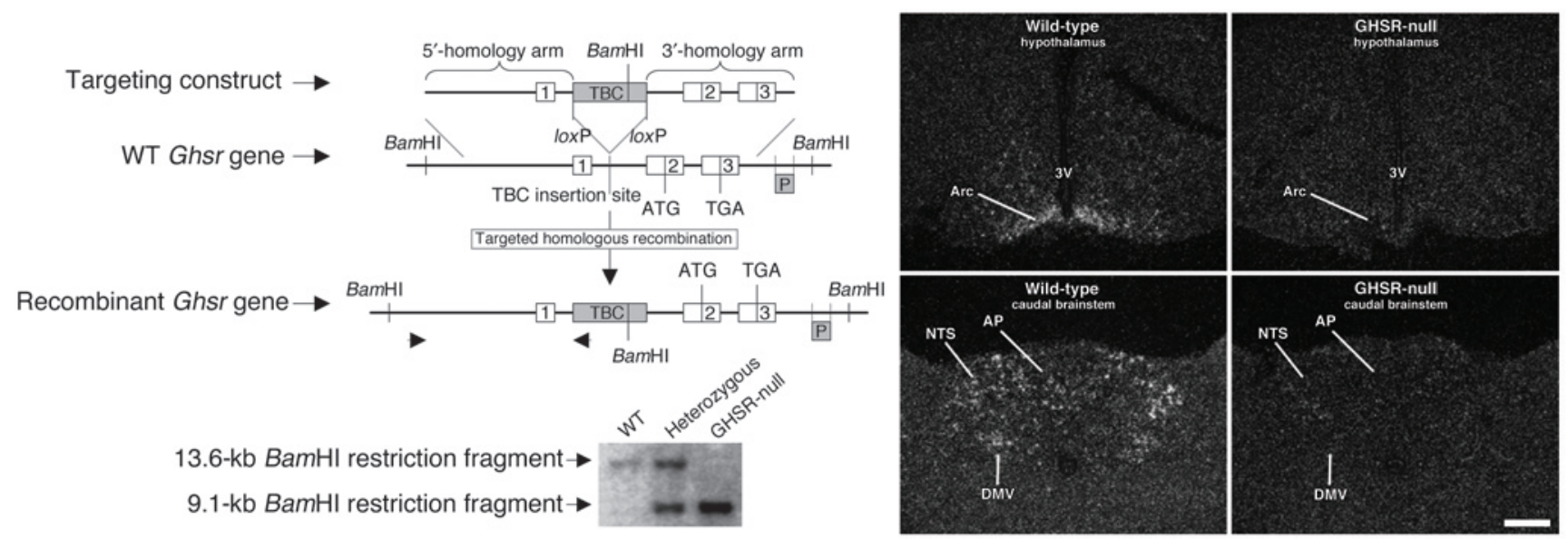

Figure 1

Gene targeting of the Ghsr locus. Upper left panel: Schematic diagram of the derivation of GHSR-null mice by homologous recombination. Lower left panel: Southern blot analysis of genomic DNA extracted from representative progeny of mating animals heterozygous for the recombinant Ghsr allele. Right panels: Representative dark-field photomicrographs of in situ hybridization histochemistry experiments performed on mouse brains using a mouse GHSR-specific riboprobe. Ghsr mRNA expression is evidenced by the white-appearing silver granules. 1-3, Ghsr exons 1-3; 3V, third ventricle; AP, area postrema; Arc, arcuate nucleus; DMV, dorsal motor nucleus of the vagus; NTS, nucleus of the solitary tract; $P$, Southern probe. Scale bar: $200 \mu \mathrm{m}$ (applies to all 4 panels).

To validate that insertion of the TBC resulted in functional GHSR-null animals, brains were examined for the presence of Ghsr mRNA by in situ hybridization histochemistry, using a riboprobe specific for mouse Ghsr mRNA. In GHSR-null mice, Ghsr transcripts were not detectable in any of the central sites that otherwise normally express GHSR, including the arcuate nucleus of the hypothalamus and the dorsal vagal complex (Figure 1). The only site in GHSR-null mice with detectable binding of the GHSR cRNA probe was the Edinger-Westphal nucleus.

We assessed the effect of a lack of functional GHSRs by monitoring acute responses to intracerebroventricular (icv) ghrelin administration. Importantly, we found that ghrelin did not stimulate food intake in GHSR-null mice, as it did in wild-type mice (Figure 2, upper panel). Furthermore, we found that arcuate nucleus neurons normally activated by ghrelin (as assessed by induction of Fos-like immunoreactivity) were no longer activated in GHSR-null mice (Figure 2, lower panels). Thus, similar to the findings in the previously reported GHSR knockout (13), our experiments confirm that GHSR is the receptor responsible for ghrelin's acute orexigenic activity.

Deletion of GHSR protects against the full development of diet-induced obesity. In order to examine the physiological significance of GHSR

\section{Figure 2}

Effects of icv administration of ghrelin to GHSR-null mice. Two-monthold female GHSR-null mice and wild-type littermates were injected icv with $2 \mu \mathrm{g}(2 \mu \mathrm{g} / \mu \mathrm{l})$ ghrelin or $1 \mu \mathrm{l}$ saline in a cross-over fashion. Upper panel: The intake of standard chow during the 2 hours immediately following the administration of ghrelin or saline. Data points and error bars correspond to the mean 2-hour food intake per body weight \pm SEM. The only significant difference among the 4 groups is noted by an asterisk; ${ }^{*} P<0.01$. Lower panels: Immunohistochemical analysis for Fos performed on tuberal hypothalamic brain sections from representative study animals following the 2-hour food intake observation period on the second drug administration day. Scale bar: $200 \mu \mathrm{m}$ (applies to all 4 panels). transcriptional blockade on long-term body weight homeostasis, we challenged GHSR-null mice and wild-type littermates to a Western-type HFD for 19 weeks, starting 1 week after weaning (when the animals were 4 weeks of age). We then followed weekly food
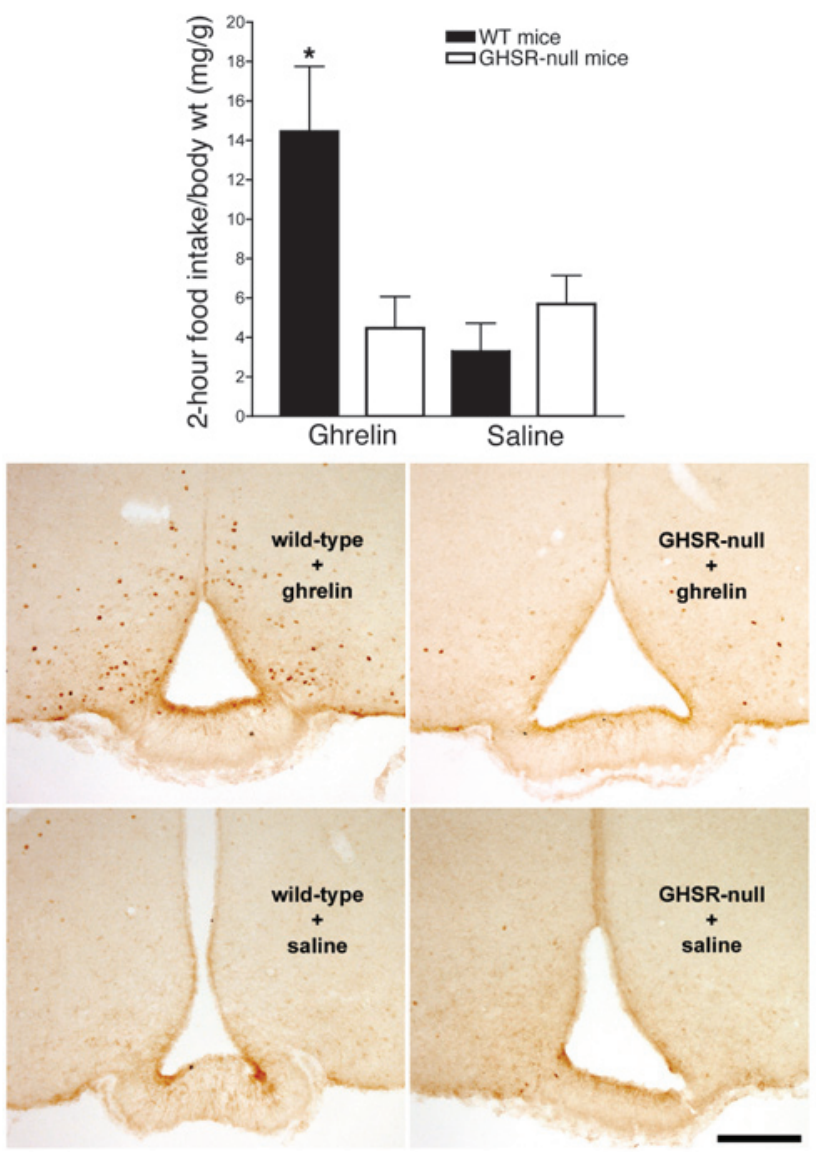

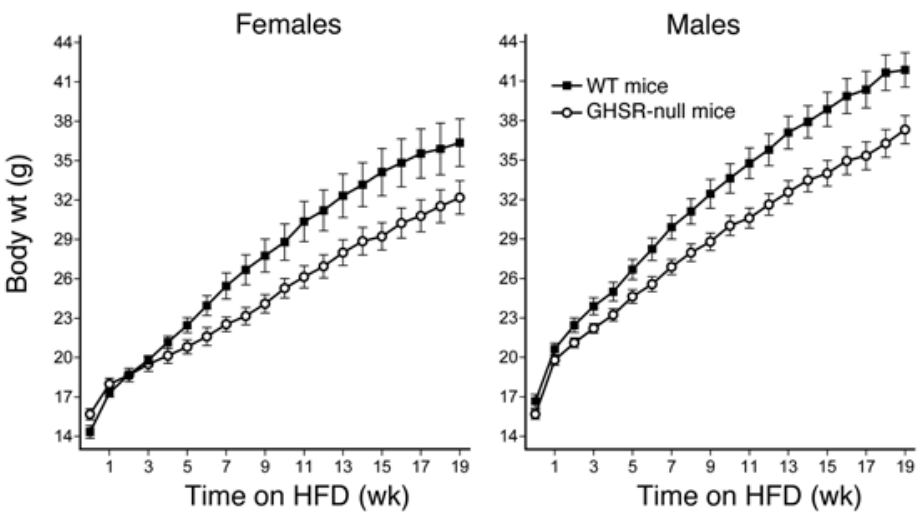

Figure 3

Body weights and body compositions of mice fed a Westerntype HFD. Weekly body weights (upper panels) of GHSR-null mice and wild-type littermates fed HFD for 19 weeks, beginning at 4 weeks of age, and their body composition at the end of the 19-week study, as determined both by carcass analysis (middle panels) and by DEXA (lower panels). Data for both female (left panels) and male (right panels) mice are presented. Data points and error bars correspond to the mean \pm SEM. Body weight and DEXA mean values were calculated from 19-22 animals per group. Carcass analysis mean values were calculated from 8-13 animals per group. Significant differences between groups noted in the body composition analyses are denoted by asterisks; ${ }^{*} P<0.05$.
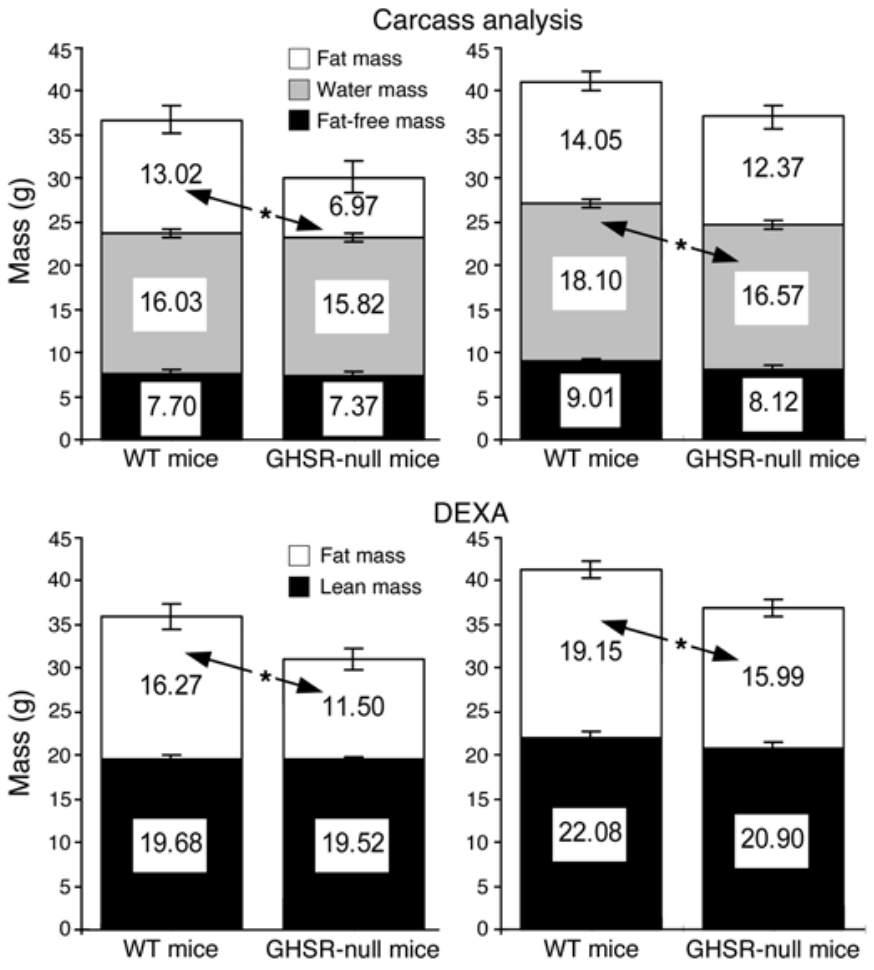

intake and weekly changes in body weight. Since a key endpoint for this study was total body fat, we also directly examined body composition of the animals. We used several different, but wellvalidated methods (dual-energy x-ray absorptiometry [DEXA], a quantitative nuclear magnetic resonance [NMR] method [ref. 18], and carcass analysis done by lyophilization and standard ether extraction [ref. 19]) to assess body composition.

At study entry, female GHSR-null mice and wild-type mice did not have statistically significant differences in body weight or body composition. However, after 8 weeks on HFD, female GHSR-null mice were significantly lighter than wild-type mice and thereafter continued to progressively diverge in body weight such that after 19 weeks, they weighed $12.7 \%$ less than wild-type mice [Figure 3; $\left.\mathrm{F}_{(19,594)}=5.84, P<0.0001\right]$. Body composition also changed over the 19-week study, such that by the study's completion, female GHSR-null mice had $46.5 \%$ less fat mass than wildtype mice, as measured by carcass analysis (Figure 3 ). Statistically significant differences in fat mass between the 2 groups at the end of the study also were observed by DEXA (Figure 3) and

NMR (data not shown). No differences were observed in lean or fat-free mass.

Male GHSR-null mice and wild-type mice also did not show statistically significant differences in body weight (Figure 3) or body composition at entry into the 19-week HFD study. As was observed for females, these 2 groups also began to progressively diverge in body weight. More specifically, by 6 weeks on HFD, male GHSR-null mice were significantly lighter than wild-type mice, and by study completion, male GHSR-null mice weighed $10.9 \%$ less than wild-type mice [Figure $\left.3 ; \mathrm{F}_{(19,735)}=6.44, P<0.0001\right]$. Furthermore, at study end, the HFD-fed male GHSR-null mice had a statistically significant reduction in fat mass (16.5\%) compared with wild-type mice when measured by DEXA (Figure 3). A subset of these male GHSR-null and wild-type mice was randomly selected to also undergo carcass (Figure 3 ) and NMR analyses, and while GHSR-null animals did display a fat mass reduction by these alternate methods, it was not statistically significant.

GHSR-null mice on HFD are bypophagic. Female GHSR-null mice on HFD manifested statistically significant reductions in mean weekly food intake (Figure 4; wild-type mice, $22.83 \pm 0.19$ g versus GHSR-null mice, $20.89 \pm 0.17 \mathrm{~g}$; $P<0.0001$ ) and cumulative food intake (wild-type mice, $434.96 \pm 12.51 \mathrm{~g}$ versus GHSR-null mice, $405.45 \pm 11.35 \mathrm{~g}$; $P<0.05)$ as compared with HFD-fed wild-type mice. Nota-

bly, the latter difference became statistically significant prior to the point of statistically significant divergence in body weight (after 6 weeks on HFD [cumulative food intake] versus after 8 weeks on HFD [body weight]). Male GHSR-null mice also demonstrated reductions in mean weekly and cumulative food intake compared with similarly fed wild-type mice (mean weekly food intake [Figure 4]: wild-type mice, $22.52 \pm 0.13$ g versus GHSR-null mice, $21.10 \pm 0.13 \mathrm{~g}, P<0.0001$; cumulative food intake: wild-type mice, $423.76 \pm 7.86 \mathrm{~g}$ versus GHSR-null mice, $402.20 \pm 7.86 \mathrm{~g}, P=0.06)$.

GHSR-null mice on HFD have lower feed and gross energetic efficiencies. Both female and male GHSR-null mice had lower feed efficiencies (20) (defined as weight gain per kilocalorie of food consumed; Figure 4; $P<0.05$ ) and lower gross energetic efficiencies $(21,22)$ (defined as energy gain per kilocalorie of food consumed; Table 1). These findings suggest that the body weights of the GHSR-null mice and, in particular, the sizes of their stored energy depots, increased at a much lower rate when compared with those of the wild-type mice on a per kilocalorieof-food-consumed basis. 

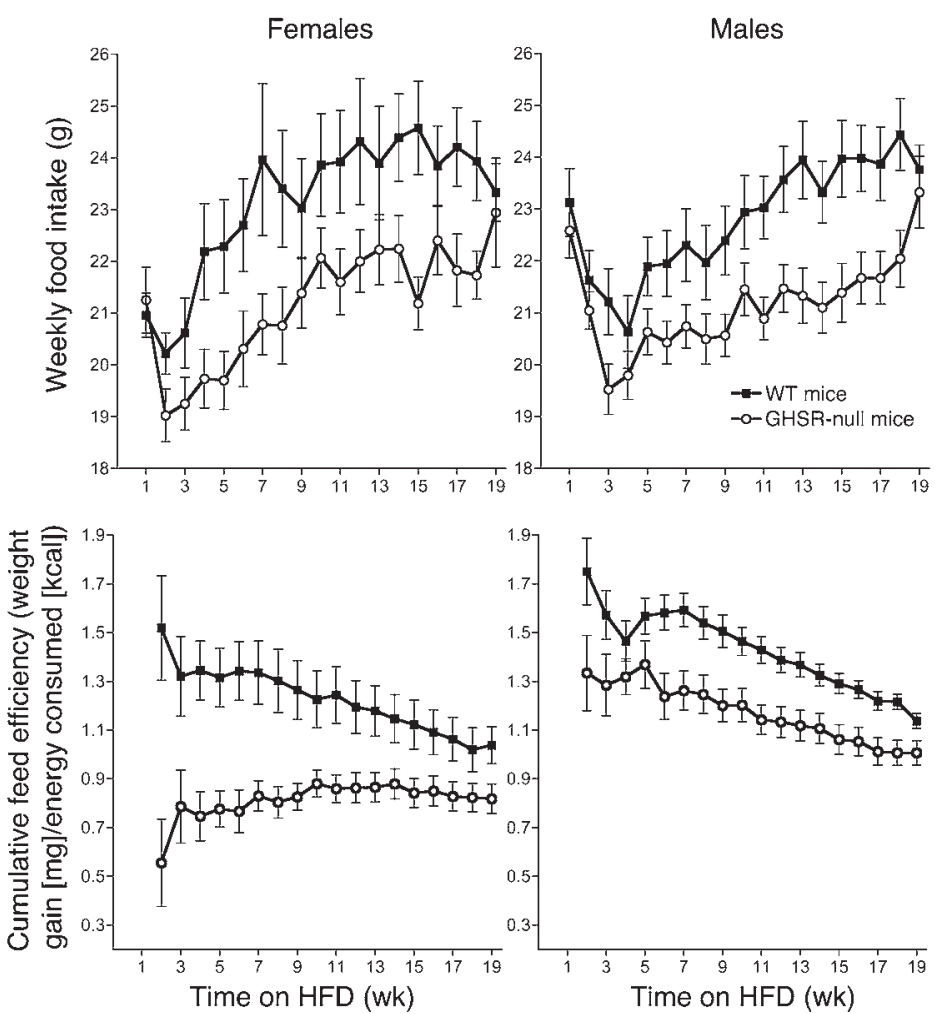

\section{Figure 4}

Weekly food intake and cumulative feed efficiency of mice fed a Western-type HFD. Weekly amounts of food intake (upper panels) and cumulative feed efficiencies (lower panels) of female (left panels) and male (right panels) GHSR-null mice and wild-type littermates fed HFD for 19 weeks, beginning at 4 weeks of age. Data points and error bars correspond to the mean \pm SEM. Mean values were calculated from 19-22 animals per group.

time point during a 19-week observation and also did now show any statistically significant differences in body fat or lean mass (Figure 6).

The effect of GHSR deletion on body length and IGF-1 levels. The documented effects of ghrelin and ghrelin mimetics on GH secretion (1) suggest that lack of ghrelin signaling may affect 1 or more components of the GH axis. However, we observed either no or only modest differences in body length between GHSR-null mice and same-sex, agematched, and diet-matched wild-type mice. In particular, we did not observe any statistically significant differences in nose-to-anus length between GHSR-null mice and wild-type littermates of either sex after HFD for 3 weeks. Female GHSR-null mice on the HFD for 19 weeks were of mean body length equal to that of similarly treated female wild-type mice. Female GHSR-null mice fed standard chow for 19 weeks were only modestly shorter (wildtype mice, $n=17,9.61 \pm 0.06 \mathrm{~cm}$ versus GHSR-null mice, $n=17,9.25 \pm 0.06 \mathrm{~cm} ; P<0.05)$. Similarly, we found only modest differences in nose-to-anus length between male GHSR-null mice and wild-type mice fed a standard chow diet for 19 weeks (wildtype mice, $n=18,9.79 \pm 0.08 \mathrm{~cm}$ versus GHSR-null mice, $n=21$, $9.60 \pm 0.08 \mathrm{~cm} ; P=0.09$ ) and those fed HFD for 19 weeks (wildtype mice, $9.54 \pm 0.07 \mathrm{~cm}$ versus GHSR-null mice, $9.22 \pm 0.07 \mathrm{~cm}$; $P<0.05)$. Interestingly, no statistically significant differences in serum IGF-1 levels were observed between genotypes for any of the HFD-fed groups (Table 2; $P>0.1$; IGF-1 levels were not determined for the animals fed standard chow).
GHSR-null mice on HFD have reduced respiratory quotients and locomotor activity. Male mice that had been on HFD for 19 weeks were placed in a Columbus Instruments Comprehensive Lab Animal Monitoring System (CLAMS) in order to assess any differences in oxygen consumption, respiratory quotient, or locomotor activity. No statistically significant differences between GHSR-null mice and wild-type mice were observed for oxygen consumption. However, GHSR-null mice did demonstrate statistically significant reductions in both respiratory quotient and locomotor activity (Figure 5). These differences were most notable during the dark cycle.

The effect of GHSR deletion in animals fed standard chow. Separate cohorts of mice were fed standard chow instead of HFD. Female GHSR-null mice became significantly lighter than wild-type controls beginning after 12 weeks on standard chow and continued to diverge in body weight such that after 19 weeks, they weighed $11.7 \%$ less than wild-type mice [Figure 6; $\left.\mathrm{F}_{(19,620)}=5.16, P<0.0001\right]$. Also, after 19 weeks, female GHSR-null mice had $35.6 \%$ less body fat than wild-type mice $(P=0.087)$ but showed no statistically significant differences in lean mass (Figure 6). Male GHSR-null and wild-type mice fed standard chow did not differ in body weight at any weekly
Table 1

Energy balance in animals fed HFD for 19 weeks

\begin{tabular}{|c|c|c|c|c|c|}
\hline Study group & $\begin{array}{l}\text { Initial body } \\
\text { energy content }{ }^{A} \\
\text { (kcal) }\end{array}$ & $\begin{array}{l}\text { Final body } \\
\text { energy content } \\
\text { (kcal) }\end{array}$ & $\begin{array}{l}\text { Body energy } \\
\text { content gainc } \\
\text { (kcal) }\end{array}$ & $\begin{array}{l}\text { Energy } \\
\text { intake }^{D} \\
\text { (kcal) }\end{array}$ & $\begin{array}{c}\text { Gross energetic } \\
\text { efficiency } \\
(\%)\end{array}$ \\
\hline Female WT mice & $64 \pm 4$ & $209 \pm 18$ & $145 \pm 16$ & $1970 \pm 57$ & $7.1 \pm 0.7$ \\
\hline Female GHSR-null mice & $71 \pm 4$ & $154 \pm 19 F$ & $83 \pm 19 F$ & $1837 \pm 51^{G}$ & $4.5 \pm 0.9^{F}$ \\
\hline Male WT mice & $77 \pm 3$ & $235 \pm 11$ & $158 \pm 11$ & $1920 \pm 36$ & $8.1 \pm 0.4$ \\
\hline Male GHSR-null mice & $80 \pm 3$ & $208 \pm 12$ & $129 \pm 13^{G}$ & $1822 \pm 36^{G}$ & $6.9 \pm 0.5^{G}$ \\
\hline
\end{tabular}

AInitial body energy content is defined as the energy stored in the fat and lean tissue of 4-week-old mice, as calculated using NMR-determined fat mass and lean body mass levels and the energy densities of fat ( $9 \mathrm{kcal} / \mathrm{g})$ and protein $(4 \mathrm{kcal} / \mathrm{g})$. BFinal body energy content is defined as the energy stored in the fat and lean tissue of a separate cohort of 23-week-old mice following a 19-week-long exposure to HFD, as calculated using NMR-determined fat mass and lean body mass levels and the energy densities of fat and protein. CBody energy content gain is defined as the difference between the final body energy content for each animal in the HFD study and the average energy content determined for the respective 4-week-old cohort. DEnergy intake is defined as the kilocalories of HFD eaten cumulatively during the 19-week study by the animals whose body compositions were assessed by NMR and was calculated by multiplying the grams of HFD consumed by the energy density $(5.3 \mathrm{kcal} / \mathrm{g})$ of the diet. EGross energetic efficiency is defined as the increase in stored body energy content per kilocalorie of food consumed (body energy content gain $\times 100 /$ energy intake). FStatistically lower value than that for wild-type controls; $P<0.05$. GStatistically lower value (trend) than that for wild-type controls; $0.05 \leq P<0.1$. 

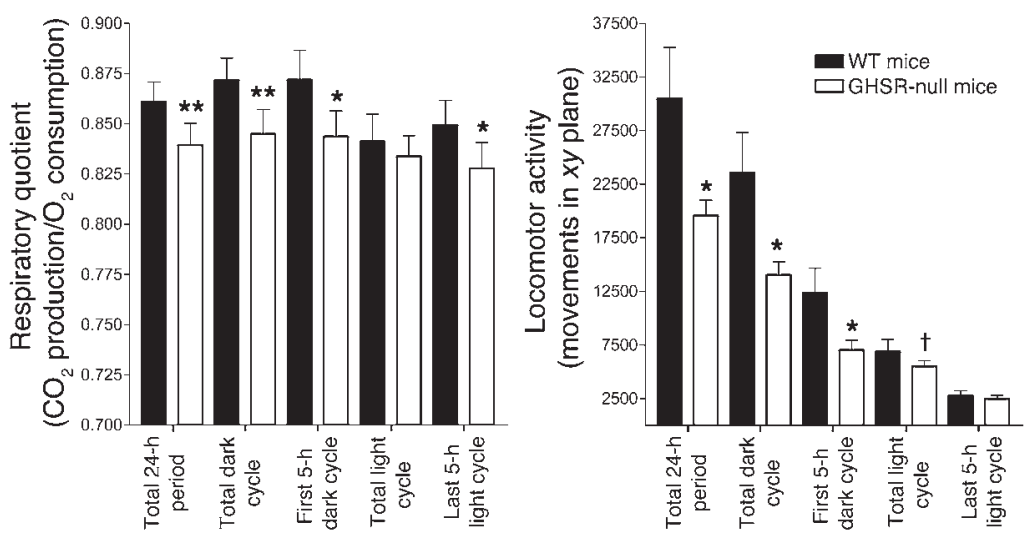

\section{Figure 5}

Respiratory quotients and locomotor activity of mice fed a Western-type HFD. Respiratory quotient (left panel) and locomotor activity (right panel) were determined for a cohort of 8 male GHSR-null mice and 8 of their male wild-type littermates, after they had been fed HFD for 19 weeks, by use of a Columbus Instruments CLAMS. Data points and error bars correspond to the mean values \pm SEM gathered over 48 hours. Statistically significant differences between GHSR-null mice and wild-type mice are indicated by asterisks; ${ }^{\star} P<0.05 ;{ }^{\star \star} P<0.005$. Statistical trends are denoted by a cross; ${ }^{\dagger} P=0.054$.
Circulating leptin levels are unchanged in GHSR-null mice. Despite the observed reduction in fat mass for the female and male GHSR-null mice on HFD, both female and male GHSR-null mice had leptin values comparable to those of their wild-type counterparts (Table 2). Similar leptin values between GHSR-null mice and wild-type mice also were demonstrated for males on standard chow for 19 weeks and for animals on HFD for 3 weeks (Table 2).

GHSR-null mice have improved blood glucose homeostasis. Blood glucose levels were randomly checked in the male mice after 19 weeks on standard chow. Despite the GHSR-null mice having final body weights and body compositions similar to those of their wild-type counterparts, their blood glucose levels were significantly lower than those of the wild-type mice (Table 3). This discrepancy was most evident when blood glucose levels were assessed toward the end of the light cycle. Corresponding serum insulin levels also were determined and were found to be lower than, although not statistically different from, those of wild-type controls (except for the animals assessed within 5 hours of the start of the light cycle, when this reduction did reach statistical significance; Table 3).

\section{Discussion}

We have generated a novel GHSR-null mouse by insertion of a TBC into the endogenous Ghsr locus. As expected, we showed that GHSR-null mice do not express Ghsr mRNA. Furthermore, GHSR-null mice do not respond to exogenous ghrelin, as assessed by their failure to acutely increase food intake or induce Fos expression in the arcuate nucleus. Thus, similar to the findings by Sun and colleagues with their GHSR-knockout animal (13), our experiments demonstrate that GHSR is the sole receptor responsible for ghrelin's acute orexigenic activity.

Resistance to diet-induced obesity. Perhaps the most striking and novel finding in our studies was the response of GHSR-null

\section{Figure 6}

Body weights and body composition of mice maintained on a standard chow diet. Weekly body weights (upper panels) and body compositions (lower panels; as determined by DEXA) of female (left panels) and male (right panels) GHSR-null mice and wild-type littermates included in a 19-week-long standard chow diet study, beginning at 4 weeks of age. Data points and error bars correspond to the mean \pm SEM. Mean values were calculated from 17-21 animals (for body weight) or 13-21 animals (for DEXA) per each group. Statistical trends are denoted by a cross; ${ }^{\dagger} P=0.087$. mice to a challenge with a Western-type, calorie-dense diet. Indeed, our results suggest that ghrelin signaling is required for the full development of diet-induced obesity. In particular, although both female and male GHSR-null mice had mean body weights and body compositions comparable to those of their same-sex wildtype littermates when measured 1 week after weaning, 19 weeks of exposure to HFD resulted in significantly less accumulation of both body weight and body fat content in GHSR-null mice as compared with littermate controls. These changes were especially prominent in female GHSR-null mice, which developed an average fat mass that was nearly $50 \%$ less than that of wild-type littermates. Furthermore, there was preservation of lean body mass. Although our results are in contrast to what has been reported in the initial characterization studies of ghrelin-knockout mice (12), we speculate that several factors may explain this apparent discrepancy. These include differences in duration of HFD exposure, animal age at time of initial HFD challenge, fat content of the food,
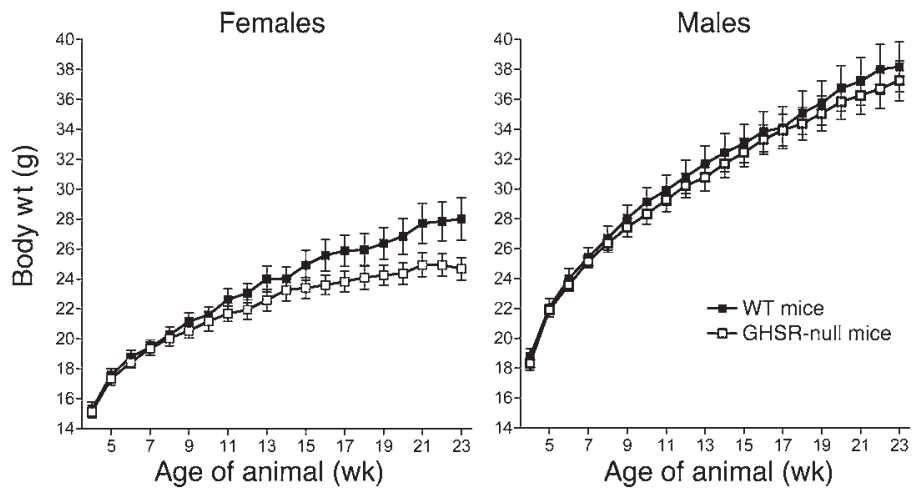

Body composition (DEXA)

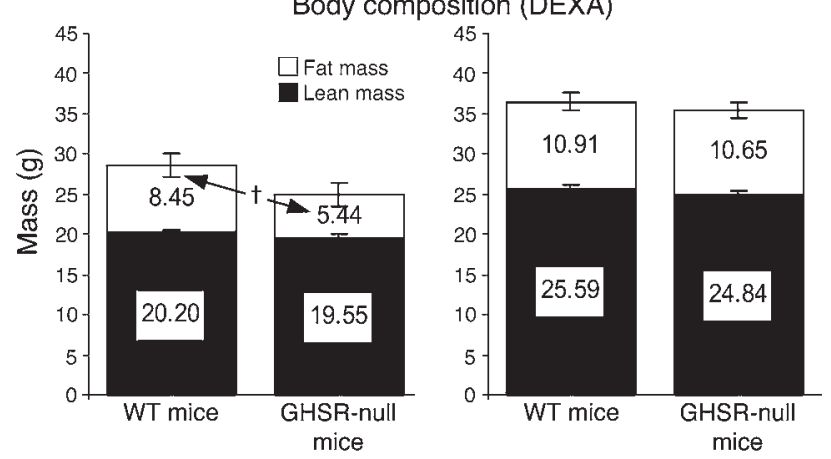


Table 2

Serum IGF-1 and leptin levels of animals on HFD

\begin{tabular}{lccc} 
Study group & Duration & IGF-1 $(\mathbf{n g} / \mathbf{m l})$ & Leptin $(\mathbf{n g} / \mathbf{m l})$ \\
Male WT mice & $19 w k$ & $592.7 \pm 34.1$ & $66.9 \pm 8.8$ \\
Male GHSR-null mice & $19 w k$ & $570.5 \pm 30.5$ & $67.4 \pm 7.9$ \\
Female WT mice & 19 wk & $508.8 \pm 33.3$ & $42.8 \pm 7.6$ \\
Female GHSR-null mice & $19 w k$ & $475.9 \pm 26.7$ & $53.6 \pm 6.7$ \\
Male WT mice & 3 wk & $588.7 \pm 21.5$ & $26.1 \pm 5.0$ \\
Male GHSR-null mice & 3 wk & $535.2 \pm 28.2$ & $26.3 \pm 5.2$ \\
Female WT mice & 3 wk & $395.4 \pm 30.2$ & $13.4 \pm 2.9$ \\
Female GHSR-null mice & 3 wk & $407.3 \pm 31.8$ & $16.7 \pm 3.1$ \\
\hline
\end{tabular}

genetic background of the animals, and study subject numbers. Importantly, our results are now complemented and supported by the findings of Wortley and colleagues in their accompanying study of ghrelin-knockout mice (23). More specifically, Wortley and colleagues show that a more prolonged and earlier exposure of ghrelin-knockout mice to HFD resulted in a mean body weight and mean body fat percentage that were lower than those of similarly treated wild-type controls (23). It should also be noted that the ghrelin-knockout mice described by Sun and colleagues had a significantly lower percentage of body fat than wild-type mice, despite the lack of divergent body weights (12).

Sexual dimorphism of responses to GHSR deletion. Of note, we found that the gender of the animals appears to influence the effectiveness of GHSR deletion at limiting body weight and body fat accumulation. For instance, not only did female GHSR-null animals accumulate less body weight and less adiposity on HFD, but a similar effect also was observed in female animals fed standard chow. This reduction in body weight on standard chow was similar to that observed previously in GHSR-knockout mice and in transgenic rats with attenuated GHSR expression $(13,24)$. In our study, the reduced body weight phenotype appeared to be mainly the result of changes in body fat, since age-matched female wildtype and GHSR-null animals fed either HFD or standard chow for 19 weeks all had equivalent amounts of lean mass but had different amounts of body fat. Female GHSR-null mice fed HFD for 19 weeks, however, did gain more weight than standard chow-fed female wild-type mice, thus suggesting that in females, deletion of GHSR does not completely prevent the obesity induced by HFD. In comparison, only male GHSR-null mice fed HFD for 19 weeks, but not male GHSR-null mice fed standard chow, accumulated less body weight than their similarly fed male wild-type counterparts. Also unlike females, male GHSRnull mice fed HFD did not gain more weight than standard chow-fed male wild-type mice. However, despite attaining similar mean final body weights, the body composition of HFD-fed male GHSR-null mice included more body fat and less lean mass than the standard chow-fed male wild-type mice.

Thus, if these sexually dimorphic differences in the responses to changes in ghrelin signaling were to hold true

\section{Table 3} trols; $0.05 \leq P<0.1$. in humans, one might predict different outcomes in males and females treated with a drug targeting GHSR. For instance, it might be predicted that females using such pharmacological interference with ghrelin signaling pathways as a preventive method against the development of obesity would not need to reduce the fat content of their diets in order to prevent unwanted body weight and body fat accumulation. Nonetheless, one might predict from our data that such a reduction in dietary fat content would enhance the drug's effectiveness in females. One might also predict that for males, such therapy would only be effective in preventing body weight and fat accumulation in individuals consuming a HFD on a regular basis, as opposed to those eating diets with a lower fat content. Furthermore, one might predict that although such therapy would cause males on a diet high in fat to attain a body weight similar to that in untreated males eating a diet with a lower fat content, this would occur at the expense of a reduction in lean mass percentage.

Mechanisms of resistance to diet-induced obesity. We believe that several processes are contributing to the resistance to diet-induced obesity demonstrated in our GHSR-null mice. One mechanism involves a reduced ability of the HFD to increase calorie consumption in GHSR-null mice. Indeed, we found this relative hypophagia in both female and male GHSR-null mice to be present within the first weeks of starting the HFD and to persist throughout the duration of the study.

Our findings also suggest that alterations in energy expenditure may potentiate the effects of hypophagia in reducing fat storage in GHSR-null mice. In particular, both female and male GHSR-null mice were shown to have lower feed efficiencies and lower gross energetic efficiencies as compared with their same-sex wild-type littermate controls. Thus, GHSR-null mice on HFD were shown to incorporate less of their consumed food into their body energy stores than wild-type mice. In the absence of any differences in the ability of GHSR-null mice to digest and absorb the HFD, these observations suggest that increased energy expenditure in GHSRnull mice contributes to their lean phenotype (21).

Interestingly, despite the observation of increased energy expenditure, the leaner GHSR-null mice did not have increased locomotor activity as compared with wild-type controls. In fact, they demonstrated a $41.7 \%$ reduction in daily locomotor activity. This may be counterintuitive based on a recent report in which ghrelin administration to rats decreased locomotor activity (25). Also, our finding of reduced locomotor activity is opposite to that found by

Blood glucose levels and corresponding serum insulin levels of male GHSR-null mice and wild-type mice after 19 weeks on a standard chow diet

\begin{tabular}{|c|c|c|c|c|}
\hline Study group & $\begin{array}{l}\text { During } \\
\text { light cycle }\end{array}$ & $\begin{array}{l}2-5 \mathrm{~h} \text { after end } \\
\text { of dark cycle }\end{array}$ & $\begin{array}{l}\text { 5-8 h after end } \\
\text { of dark cycle }\end{array}$ & $\begin{array}{l}\text { 8-11 h after end } \\
\text { of dark cycle }\end{array}$ \\
\hline \multicolumn{5}{|c|}{ Blood glucose (mg/dl) } \\
\hline WT mice & $156 \pm 5(18)^{A}$ & $159 \pm 8(6)$ & $150 \pm 6(9)$ & $171 \pm 10$ \\
\hline GHSR-null mice & $140 \pm 4(21)^{B}$ & $147 \pm 6(10)$ & $132 \pm 7(6)^{c}$ & $137 \pm 8(5)^{\mathrm{B}}$ \\
\hline \multicolumn{5}{|c|}{ Serum insulin (ng/mI) } \\
\hline WT mice & $0.778 \pm 0.115$ & $1.002 \pm 0.190$ & $0.648 \pm 0.167$ & $0.797 \pm 0.33$ \\
\hline GHSR-null mice & $0.523 \pm 0.106$ & $0.474 \pm 0.135^{B}$ & $0.476 \pm 0.205$ & $0.715 \pm 0.29$ \\
\hline
\end{tabular}

AThe number of samples tested for each study group are noted in parentheses. ${ }^{B}$ Statistically lower value than for wild-type controls; $P<0.05$. 'Statistically lower value (trend) than that for wild-type con- 
Wortley and colleagues in their study on ghrelin-knockout mice (23). We would assert that such a discrepancy between GHSR-null mice and ghrelin-knockout mice may stem, at least in part, from a potentially fundamental difference between blocking the transcription of ghrelin's receptor and blocking the transcription of ghrelin. In fact, Holst and colleagues (26) recently demonstrated that GHSR transfected into COS-7 cells and HEK 293 cells possessed high constitutive activity. Thus, blocking the transcription of a receptor with both high ligand-independent signaling and the ability to respond to increases in ghrelin could potentially have distinct, ghrelin-independent and ghrelin-dependent effects on the activity of neurons.

Decreased respiratory quotient may also play a role in the GHSRnull mice's resistance to diet-induced obesity. The observed decrease in respiratory quotient during the long-term HFD study represents a shift in metabolic fuel preference toward the utilization of fat as an energy substrate, which in turn may contribute to less accumulation of fat stores. This response was not unexpected in our mice, as Wortley and colleagues had made similar observations in their initial study with ghrelin-knockout animals (14). These results are also consistent with the findings of another study in which administration of GHSR agonist increased respiratory quotient $(10,14)$.

Finally, we believe that the degree of leptinemia in GHSR-null animals also may contribute to the obesity-resistant phenotype. Leptin is known to decrease food intake and increase energy expenditure. Thus, the higher-than-expected leptin levels observed in GHSR-null mice may contribute to their hypophagia and increased energy expenditure, as compared with wild-type mice. Although speculative, such a finding also suggests that intact GHSR signaling normally restricts leptin release, whereas deletion of GHSR, as achieved here, disinhibits leptin release.

We do not believe that differences in body length substantially contribute to the decreased body weight or changed body compositions in the GHSR-null mice. In particular, although decreases in nose-to-anus length were observed in certain of the adult GHSRnull mouse groups, these amounted to only a $1.9-3.6 \mathrm{~mm}$, or a $1.9-3.7 \%$, reduction as compared with matched wild-type animals. Of interest, Sun and colleagues' ghrelin-knockout mice and GHSR-knockout mice $(12,13)$ and Wortley and colleagues' ghrelin-knockout mice (23) were all shown to have body lengths that were indistinguishable from those of wild-type littermates.

It is also unlikely that decreases in GH secretion, which might be expected to occur under conditions of GHSR transcriptional blockade and the resultant blockade of ghrelin action, are contributing to the resistance to diet-induced obesity in GHSR-null mice. For example, we were unable to demonstrate any statistically significant differences in IGF-1 values for GHSR-null mice and wild-type in any of the cohorts tested. Furthermore, even if there had been a significant lowering of IGF-1 values in the GHSR-null mice, this would be predicted to induce both a higher percentage of body fat (which is the opposite of what we observed) and a lower percentage of lean body mass (which we also did not observe) as compared with similarly treated wild-type mice (27). Our finding of unchanged IGF-1 levels was similar to that observed by Wortley and colleagues (23) in HFD-fed ghrelin-knockout mice but was unlike that in Sun and colleagues' GHSR-knockout mice, which were found to have statistically lower IGF-1 levels than same-age (24-week-olds) and same-gender wild-type littermates (13).

Effect of GHSR deletion on glucose homeostasis. In addition to effects on body weight, GHSR deletion led to lower blood glucose lev- els in males that had been maintained on a standard chow diet. Interestingly, corresponding insulin levels for the GHSR-null mice were not increased. The insulin levels ranged in a timedependent manner within the light cycle from being statistically lower than to not statistically different from the insulin levels of wild-type controls. Because both the GHSR-null mice and wildtype mice in the long-term standard chow study finished with similar body weights and body compositions, the lower blood glucoses and low-to-normal insulin levels for the GHSR-null mice suggest that GHSR-null mice have enhanced insulin sensitivity as compared with wild-type controls.

Such findings on the involvement of ghrelin in glycemic control are consistent with previous reports $(9,28-30)$. Similar to our findings, previous studies have demonstrated that interference with ghrelin signaling by use of GHSR antagonists decreases blood glucose levels in wild-type mice, GH-deficient lit/lit mice, and $o b / o b$ mice $(9,28)$. Also supportive of our findings was a study in which nonobese healthy male subjects were administered a ghrelin mimetic under the condition of GH receptor blockade by pegvisomant (30). In these individuals, the ghrelin mimetic induced increases in both glucose and insulin levels, suggesting ghrelin mimetic-mediated, GH-independent insulin resistance. This is consistent with our findings in which GHSR transcriptional blockade led to reductions in glucose and insulin levels, without concomitant changes in IGF-1 levels. However, unlike our findings, in other studies, GHSR antagonists were shown to raise insulin levels $(9,28)$. Such studies are supported by other experiments in which exposure of isolated mouse and rat islets or isolated rat pancreas to ghrelin reduced insulin release $(29,31)$. Thus, although most studies agree that ghrelin does influence blood glucose levels, the exact mechanism by which ghrelin influences glucose homeostasis remains to be determined.

\section{Methods}

Generation and maintenance of GHSR-null mice. The targeting construct was generated using ET cloning and related technologies within EL250 cells $(32,33)$. Its assembly involved linking together DNA segments containing the elements described in Results into a TBC; these DNA segments were derived from 4 different plasmids, including pGT1.8geo (kindly provided by J. Rossant, Mount Sinai Hospital, Toronto, Ontario, Canada), pSV-Cre (kindly provided by F. Stewart, European Molecular Biology Laboratory [EMBL] Heidelberg, Heidelberg, Germany), pGL3-Control (Promega), and pHR68MAZ (kindly provided by Barry Rosen, Wellcome Trust Sanger Institute, Cambridge, United Kingdom). loxP sites were added to flank the TBC, which next was inserted into a mouse GHSRcontaining bacterial artificial chromosome (RPCI.22 494 N7; BACPAC Resources Center at Children's Hospital Oakland Research Institute), at an insertion site located 256 bp upstream of the Ghsr start codon (within a 196-bp putative intron downstream of the proposed Ghsr transcriptional start site [Celera Genomics database; Applera Corp.]). The final targeting construct, which consisted of the loxP-flanked TBC flanked by $4.5-\mathrm{kb}$ Ghs $r$ homology arms, was electroporated into ES cells, and correct targeting was confirmed by Southern blot and PCR analyses. After germline transmission was established, the chimera carrying the recombinant allele was crossed onto a C57BL/6J background to yield $\mathrm{N}_{2} \mathrm{~F}_{1}$ animals on which the experiments reported in this study were conducted. Animals were housed under 14 hours of light/10 hours of dark per day in a temperature-controlled environment. They were fed either standard chow (8664 F6 Rodent Diet; Harlan Teklad) or HFD (88137 Western diet; Harlan Teklad). Locomotor activity, respiratory quotients, and oxygen 
consumption were measured in a Columbus Instruments CLAMS by the BIDMC Animal Physiology Core. All experiments were approved by the BIDMC Institutional Animal Care and Use Committee.

Histochemical analyses. Histochemical analyses were done as previously described $(34,35)$. These involved the use of a 372-nt-long antisense riboprobe complementary to the final 117-nt of the mouse Ghsr type $1 \mathrm{mRNA}$ coding region plus the first $255 \mathrm{nt}$ of its $3^{\prime}$-untranslated region and Fos rabbit primary antiserum (1:150,000; Ab-5, Oncogene Science).

Acute food intake experiments. Sixty-day-old female study animals were implanted with indwelling icv cannulae. Afterward, animals were housed in separate cages, and correct placement of the indwelling cannulae was validated 10 days later by observing the response to icv administration of $20 \mathrm{ng}$ angiotensin II. In the second postoperative week, animals were injected with rat/mouse ghrelin (catalog 031-31; Phoenix Pharmaceuticals Inc.) or saline icv, and 2-hour intake of standard chow was measured. One week later, the procedure was repeated in a cross-over fashion, after which mice were sacrificed and brains were isolated for Fos immunohistochemical analysis.

Long-term feeding studies. GHSR-null and wild-type littermate study animals were derived from 30 breeding pairs of $\mathrm{N}_{2}$ animals heterozygotic for the recombinant GHSR allele. Animals were weaned at 3 weeks of age and were separated into 4 cohorts; these animals were weighed weekly. Each animal in the first cohort was placed in an individual cage at 4 weeks of age and was provided with ad libitum HFD and water. Weekly food intake was measured. After 19 weeks, the animals were subjected to a series of final procedures and analyses, including blood draws and body composition analysis by DEXA. DEXA was performed using the facilities of the Metabolic Physiology Core of the Diabetes Endocrinology Research Center at BIDMC. Because total body fat was a key endpoint for this study, a random subset of animals from the first cohort was shipped to the University of Cincinnati Mouse Metabolic Phenotyping Center for further body composition analysis by NMR (18) and carcass analysis (19). For several practical and scientific reasons, we were unable to independently assess the body composition of all the study animals with these 3 different methods. The next 2 cohorts were generated primarily in order to determine body composition prior to starting HFD and soon after starting HFD; these included 1 cohort of males and females weaned at 3 weeks of age, sacrificed at 4 weeks of age, and sent to Cincinnati for NMR and another cohort of males and females weaned at 3 weeks of age, housed multiply with same-sex littermates (3-4 animals per cage) beginning at 4 weeks of age, and exposed to HFD for 3 weeks, after which the animals were analyzed by DEXA and sacrificed. A fourth cohort of males and females was treated similarly, except they were housed multiply and were fed standard chow; these animals all had DEXA performed after 19 weeks; weekly food intake was not measured.

Gross energetic efficiency was determined by first estimating the energy content stored in the bodies of the mice sacrificed at 4 weeks of age and of the mice challenged with HFD for 19 weeks. This estimate involved multiplying the NMR-determined body fat and lean mass levels by the energy densities of fat and protein, respectively, and necessitated the conservative assumptions that each gram of body fat mass was composed of 1 gram of fat and that each gram of body lean mass was composed of 1 gram of protein. Next, the average energy content determined for each 4-week-old subgroup was subtracted from the energy content determined for each of the appropriate same-sex, same-genotype animals on HFD for 19 weeks, thus yielding an energy gain value for each 19-week-HFD-fed animal. The energy content of the HFD consumed by each mouse from age 4 weeks until the end of the 19-week HFD study was determined using the energy density of the HFD $(5.3 \mathrm{kcal} / \mathrm{g})$. The calculation of feed efficiency over time was determined by comparing body weight at each weekly time point to the body weight after 1 week on HFD and then dividing this body weight gain value by the energy content of the HFD consumed over the same duration.

Hormone analyses. Reagents used included: Active Mouse/Rat IGF-I EIA Kit (Diagnostic Systems Laboratories Inc.), Mouse Leptin ELISA Kit (Crystal Chem, Inc.), Ultra Sensitive Rat Insulin ELISA Kit (Crystal Chem Inc.), and Mouse Insulin Standard 1.28 ng (Crystal Chem Inc.). Assays were performed in duplicate.

Statistics. Data are expressed as mean \pm SEM. Comparisons were carried out by repeated-measures ANOVA when assessing the acute food intake responses (with genotype as "between factor" and treatment as "within factor" variables). Repeated-measures ANOVA also was used to compare feed efficiencies and body weight changes over time. For the body weight comparisons, 3 independent analyses were performed for each sex, including 1 in which the effects of genotype ("between factor" variable) and week of study ("within factor" variable) were assessed for animals on HFD; a second in which the effects of genotype and week of study were assessed for animals on standard chow; and a third in which both genotype and diet were used as "between factor" variables and week of study as the "within factor" variable. One-way ANOVA or 2-way ANOVA analyses were performed when assessing the effects of genotype alone or both genotype and diet on all other parameters. Tukey-Kramer post-hoc analysis was used for all comparisons with significant $P$ values. $P<0.05$ was considered statistically significant. $0.05 \leq P<0.1$ was considered to be evident of statistical trends. The repeated-measures ANOVA statistics as presented include not only the $P$ value, but also its corresponding $F$ ratio value and, in parentheses, the degrees of freedom of the numerator and denominator used to calculate the F ratio. The program NCSS 2004 (Number Cruncher Statistical Systems) was used for all statistical analyses.

\section{Acknowledgments}

We thank A. Pullen and J. Yu for excellent technical assistance, N. Copeland for providing EL250 cells, B. Rosen for providing the pHR68MAZ plasmid, J. Rossant for providing the pGT1.8geo plasmid, F. Stewart for providing the pSV-Cre plasmid, L. Keller and K. Smith of the University of Cincinnati Mouse Metabolic Phenotyping Center for performing the carcass and NMR body composition analyses, and E. Flier and F. Marino of the BIDMC Animal Physiology Core for performing the CLAMS analyses. This work was supported by funding from the NIH (1F32DK64564-01, 5T32DK07516, DK53301, DK56116, DK59630), the Metabolic Physiology Core of the Diabetes Endocrinology Research Center (NIDDK P30 DK57521), a Boston Obesity Nutrition Research Center Pilot Project Award, an Endocrine Fellows Foundation Research Grant, and Takeda Chemical Industries.

Received for publication June 20, 2005, and accepted in revised form October 4, 2005.

Address correspondence to: Joel K. Elmquist or Bradford B. Lowell, Department of Medicine, Division of Endocrinology, Diabetes and Metabolism, Beth Israel Deaconess Medical Center, 99 Brookline Avenue, RN-343, Boston, Massachusetts 02215, USA. Phone: (617) 667-0845; Fax: (617) 667-2927; E-mail: jelmquis@bidmc.harvard. edu (J.K. Elmquist). Phone: (617) 667-5954; Fax: (617) 667-3304; E-mail: blowell@bidmc.harvard.edu (B.B. Lowell).

Bradford B. Lowell and Joel K. Elmquist contributed equally to this work. 
1. Kojima, M., et al. 1999. Ghrelin is a growth-hormone-releasing acylated peptide from stomach. Nature. 402:656-660.

2. Cummings, D.E., et al. 2001. A preprandial rise in plasma ghrelin levels suggests a role in meal initiation in humans. Diabetes. 50:1714-1719.

3. Cummings, D.E., et al. 2002. Plasma ghrelin levels after diet-induced weight loss or gastric bypass surgery. N. Engl. J. Med. 346:1623-1630.

4. Tschop, M., Smiley, D.L., and Heiman, M.L. 2000. Ghrelin induces adiposity in rodents. Nature. 407:908-913.

5. Nakazato, M., et al. 2001. A role for ghrelin in the central regulation of feeding. Nature. 409:194-198.

6. Asakawa, A., et al. 2001. Ghrelin is an appetite-stimulatory signal from stomach with structural resemblance to motilin. Gastroenterology. 120:337-345.

7. Kamegai, J., et al. 2000. Central effect of ghrelin, an endogenous growth hormone secretagogue, on hypothalamic peptide gene expression. Endocrinology. 141:4797-4800.

8. Yasuda, T., Masaki, T., Kakuma, T., and Yoshimatsu, H. 2003. Centrally administered ghrelin suppresses sympathetic nerve activity in brown adipose tissue of rats. Neurosci. Lett. 349:75-78.

9. Asakawa, A., et al. 2003. Antagonism of ghrelin receptor reduces food intake and body weight gain in mice. Gut. 52:947-952.

10. Tschop, M., Statnick, M.A., Suter, T.M., and Heiman, M.L. 2002. GH-releasing peptide- 2 increases fat mass in mice lacking NPY: indication for a crucial mediating role of hypothalamic agouti-related protein. Endocrinology. 143:558-568.

11. Wren, A.M., et al. 2001. Ghrelin causes hyperphagia and obesity in rats. Diabetes. 50:2540-2547.

12. Sun, Y., Ahmed, S., and Smith, R.G. 2003. Deletion of ghrelin impairs neither growth nor appetite. Mol. Cell. Biol. 23:7973-7981.

13. Sun, Y., Wang, P., Zheng, H., and Smith, R.G. 2004. Ghrelin stimulation of growth hormone release and appetite is mediated through the growth hormone secretagogue receptor. Proc. Natl. Acad. Sci.
U. S. A. 101:4679-4684.

14. Wortley, K.E., et al. 2004. Genetic deletion of ghrelin does not decrease food intake but influences metabolic fuel preference. Proc. Natl. Acad. Sci. U. S. A. 101:8227-8232.

15. Ashfield, R., et al. 1994. MAZ-dependent termination between closely spaced human complement genes. EMBO J. 13:5656-5667.

16. Joyner, A.L., Skarnes, W.C., and Rossant, J. 1989 Production of a mutation in mouse En- 2 gene by homologous recombination in embryonic stem cells. Nature. 338:153-156.

17. Yonaha, M., and Proudfoot, N.J. 1999. Specific transcriptional pausing activates polyadenylation in a coupled in vitro system. Mol. Cell. 3:593-600.

18. Taicher, G.Z., Tinsley, F.C., Reiderman, A., and Heiman, M.L. 2003. Quantitative magnetic resonance (QMR) method for bone and whole-body-composition analysis. Anal. Bioanal. Chem. 377:990-1002.

19. Strader, A.D., Reizes, O., Woods, S.C., Benoit, S.C., and Seeley, R.J. 2004. Mice lacking the syndecan-3 gene are resistant to diet-induced obesity. J. Clin. Invest. 114:1354-1360. doi:10.1172/ JCI200420631.

20. Chen, A.S., et al. 2000. Inactivation of the mouse melanocortin-3 receptor results in increased fat mass and reduced lean body mass. Nat. Genet. 26:97-102.

21. Picard, F., Deshaies, Y., Lalonde, J., Samson, P., and Richard, D. 2000. Topiramate reduces energy and fat gains in lean $(\mathrm{Fa} /$ ?) and obese $(\mathrm{fa} / \mathrm{fa})$ Zucker rats. Obes. Res. 8:656-663.

22. Langley, S.C., and York, D.A. 1990. Effects of antiglucocorticoid RU 486 on development of obesity in obese fa/fa Zucker rats. Am. J. Physiol. 259:R539-R544.

23. Wortley, K.E., et al. 2005. Absence of ghrelin protects against early-onset obesity. J. Clin. Invest. 115:3573-3578. doi:10.1172/JCI26003.

24. Shuto, Y., et al. 2002. Hypothalamic growth hormone secretagogue receptor regulates growth hormone secretion, feeding, and adiposity. J. Clin. Invest.
109:1429-1436. doi:10.1172/JCI200213300.

25. Tang-Christensen, M., et al. 2004. Central administration of ghrelin and agouti-related protein (83-132) increases food intake and decreases spontaneous locomotor activity in rats. Endocrinology. 145:4645-4652.

26. Holst, B., Cygankiewicz, A., Jensen, T.H., Ankersen, M., and Schwartz, T.W. 2003. High constitutive signaling of the ghrelin receptor - identification of a potent inverse agonist. Mol. Endocrinol. 17:2201-2210.

27. Jorgensen, J.O., et al. 1994. Adult growth hormone deficiency. Horm. Res. 42:235-241.

28. Dezaki, K., et al. 2004. Endogenous ghrelin in pancreatic islets restricts insulin release by attenuating $\mathrm{Ca} 2+$ signaling in beta-cells: implication in the glycemic control in rodents. Diabetes. 53:3142-3151.

29. Broglio, F., et al. 2001. Ghrelin, a natural GH secretagogue produced by the stomach, induces hyperglycemia and reduces insulin secretion in humans. J. Clin. Endocrinol. Metab. 86:5083-5086.

30. Muller, A.F., et al. 2001. Blockade of the growth hormone $(\mathrm{GH})$ receptor unmasks rapid GH-releasing peptide-6-mediated tissue-specific insulin resistance. J. Clin. Endocrinol. Metab. 86:590-593.

31. Egido, E.M., Rodriguez-Gallardo, J., Silvestre, R.A., and Marco, J. 2002. Inhibitory effect of ghrelin on insulin and pancreatic somatostatin secretion. Eur. J. Endocrinol. 146:241-244.

32. Muyrers, J.P., Zhang, Y., and Stewart, A.F. 2001. Techniques: recombinogenic engineering - new options for cloning and manipulating DNA. Trends Biochem. Sci. 26:325-331.

33. Lee, E.C., et al. 2001. A highly efficient Escherichia coli-based chromosome engineering system adapted for recombinogenic targeting and subcloning of BAC DNA. Genomics. 73:56-65.

34. Marcus, J.N., et al. 2001. Differential expression of orexin receptors 1 and 2 in the rat brain. J. Comp. Neurol. 435:6-25.

35. Kishi, T., et al. 2003. Expression of melanocortin 4 receptor mRNA in the central nervous system of the rat. J. Comp. Neurol. 457:213-235. 